\title{
Phytophthora Database: A Forensic Database Supporting the Identification and Monitoring of Phytophthora
}

Jongsun Park, School of Agricultural Biotechnology and Fungal Bioinformatics Laboratory, Seoul National University, Seoul, Korea; Bongsoo Park, Department of Plant Pathology, and Narayanan Veeraraghavan, Center for Computational Genomics, Pennsylvania State University, University Park 16802; Kyongyong Jung and Yong-Hwan Lee, School of Agricultural Biotechnology and Fungal Bioinformatics Laboratory, Seoul National University; Jaime E. Blair, David M. Geiser, Scott Isard, Michele A. Mansfield, Ekaterina Nikolaeva, and Sook-Young Park, Department of Plant Pathology, Pennsylvania State University; Joseph Russo, ZedX, Inc., Bellefonte, PA 16823; Seong H. Kim, Pennsylvania Department of Agriculture, Harrisburg 17110; Matthew Greene and Kelly L. Ivors, Mountain Horticultural Crops Research \& Extension Center, North Carolina State University, Fletcher 28732; Yilmaz Balci, Division of Plant \& Soil Sciences, West Virginia University, Morgantown 26506; Masoomeh Peiman, Donald C. Erwin, and Michael D. Coffey, Department of Plant Pathology, University of California, Riverside 92521; Amy Rossman, David Farr, and Erica Cline, United States Department of Agriculture-Agricultural Research Service (USDA-ARS), Systematic Botany \& Mycology Laboratory, Beltsville, MD 20705; Niklaus J. Grünwald, USDA-ARS Horticultural Crops Research Laboratory, Corvallis, OR 97330; Douglas G. Luster, USDA-ARS, Foreign Disease-Weed Science Research Unit, Ft. Detrick, MD 21702; Julia Schrandt and Frank Martin, USDA-ARS, Salinas, CA 93906; Olaf K. Ribeiro, Ribeiro Plant Lab, Inc., Bainbridge Island, WA 98110; and Izabela Makalowska, Center for Computational Genomics, and Seogchan Kang, Department of Plant Pathology, Pennsylvania State University

\begin{abstract}
Park, J., Park, B., Veeraraghavan, N., Jung, K., Lee, Y.-H., Blair, J. E., Geiser, D. M., Isard, S., Mansfield, M. A., Nikolaeva, E., Park, S.-Y., Russo, J., Kim, S. H., Greene, M., Ivors, K. L., Balci, Y., Peiman, M., Erwin, D. C., Coffey, M. D., Rossman, A., Farr, D., Cline, E., Grünwald, N. J., Luster, D. G., Schrandt, J., Martin, F., Ribeiro, O. K., Makalowska, I., and Kang, S. 2008. Phytophthora Database: A forensic database supporting the identification and monitoring of Phytophthora. Plant Dis. 92:966-972.

Phytophthora spp. represent a serious threat to agricultural and ecological systems. Many novel Phytophthora spp. have been reported in recent years, which is indicative of our limited understanding of the ecology and diversity of Phytophthora spp. in nature. Systematic cataloging of genotypic and phenotypic information on isolates of previously described species serves as a baseline for identification, classification, and risk assessment of new Phytophthora isolates. The Phytophthora Database (PD) was established to catalog such data in a web-accessible and searchable format. To support the identification of new Phytophthora isolates via comparison of their sequences at one or more loci with the corresponding sequences derived from the isolates archived in the PD, we generated and deposited sequence data from more than 1,500 isolates representing the known diversity in the genus. Data search and analysis tools in the PD include BLAST, Phyloviewer (a program for building phylogenetic trees using sequences of selected isolates), and Virtual Gel (a program for generating expected restriction patterns for given sequences). The PD also provides a customized means of storing and sharing data via the web. The PD serves as a model that easily can be adopted to develop databases for other important pathogen groups.
\end{abstract}

Additional keywords: culture collection, disease management, invasive pathogens, molecular diagnosis

Pathogens frequently migrate from one region to another via global trade, human travel, and weather-related events. In re-

Corresponding authors: S. Kang and I. Makalowska E-mail: sxk55@psu.edu and izabelam@psu.edu

The first three authors contributed equally to this work.

Accepted for publication 23 January 2008.

doi:10.1094/PDIS-92-6-0966

(c) 2008 The American Phytopathological Society cent years, the expanding volume of trade and the increasing numbers of entry points have greatly accelerated the introduction and establishment of invasive pathogens. There are numerous historical and contemporary examples of major crop loss caused by invasive pathogens (1). Invasive pathogens can be equally catastrophic in natural landscapes; the virtual extinction of the American chestnut in eastern forests and the tremendous loss of landscape elms in American cities can be attributed directly to accidental introduction of exotic fungi (14,23). Early detection, accurate identification, and the ability to trace pathogens back to their likely origin will significantly increase the probability of preventing the spread of invasive pathogens. Given the global nature of disease problems, global cooperation in mapping and documenting the diversity and distribution of pathogens is essential for disease management. Because science builds on existing knowledge, such cooperation also is important for the advancement of plant pathology. Toward the goal of enhancing global cooperation in studying and managing major pathogens, we have established a supporting cyberinfrastructure known as the Phytophthora Database (PD; http:// www.phytophthoradb.org/).

The genus Phytophthora was chosen as the first step toward the goal of building a global plant pathogen database primarily because of its practical significance. The destructive potential of members of Phytophthora is well illustrated by $P h y$ tophthora infestans; late blight has become globally problematic due to high levels of pathogen diversity and the introduction of new, fungicide-resistant lineages (12). Sudden oak death in the western United States and diseases on ornamental plants throughout the United States and Europe (30), caused by $P$. ramorum, are examples of the threat to forest ecosystems and the nursery industry. Many novel species, including $P$. ramorum, have been reported in recent years $(5,7,9,11,16,18-20,28,36)$, underscoring our limited understanding of the ecology and diversity of Phytophthora spp. in nature. Furthermore, the potential 
for new Phytophthora spp. to emerge from hybridization events exists $(4,6,8)$. The likelihood that $P$. ramorum will not be the last threat from the genus Phytophthora signifies the importance of continuously monitoring the diversity, distribution, and dynamics of Phytophthora spp. in nature. Systematic archiving of resulting cultures and data, in a readily accessible format, is necessary to maximize their utility in protecting global agricultural production and ecological systems from Phytophthora diseases. The PD offers an effective means for archiving and sharing of data and can serve as a model for cataloging other pathogen groups.

\section{MATERIALS AND METHODS}

Culture collection and growth conditions. Isolates characterized in this study are mainly archived in the World Phytophthora Collection (http://phytophthora.ucr.edu/) at the University of California-Riverside or at the Pennsylvania Department of Agriculture. Some cultures are stored in more than one location. Corresponding accession numbers of isolates in individual culture collection site are indicated in the PD. Cultures are preserved cryogenically under liquid nitrogen. For DNA extraction, actively growing cultures were produced in either clarified (1:2) V8 broth or pea broth and harvested after 4 to 10 days.

Polymerase chain reaction and sequence analysis. Genomic DNA was extracted as previously described (3). Polymerase chain reaction (PCR) conditions for amplifying target loci varied from locus to locus and are described along with the sequences and locations of the primers used in the Genetic Markers section of the PD. Amplified fragments were purified using spin columns (Qiagen) or treated with ExoSAP-IT enzyme (USB, Cleveland, $\mathrm{OH}$ ) and directly sequenced with the same primers used for PCR amplification. For certain loci, internal primers also were used to complete the sequencing. Prior to depositing resulting sequence data to the PD, they were edited manually and aligned with sequences from other isolates within the same species to further verify polymorphic sites.

Database construction. The databases were built using MySQL, which is an open-source database management program. The user interface was constructed using PHP scripts and operates from an Apache web server in the Linux system. Using ANSI-C and Perl, middleware was designed based on the Comparative Fungal Genomics Platform (http://cfgp.snu.ac.kr/) (27) to link data analysis and visualization tools to the user interface and to manage tasks, such as BLAST search and ClustalW.

\section{RESULTS}

Organization of the PD. The organization of the PD is illustrated in Figure 1.
Relational databases house phenotypic and genotypic data and literature information associated with individual species and isolates. The home page provides menus that lead users to the following: (i) an overview of the PD, including user manuals; (ii) current statistics on the stored data (e.g., numbers of species, isolates, and sequences archived in the PD), (iii) individual databases; (iv) tools for data search, analysis, and storage; and (v) recent publications and news on Phytophthora spp. Via the user interface, users can search these databases using key words (e.g., species name, genetic marker, host, reference, and so on) to retrieve desired information or marker sequences via BLAST (2) to identify a new isolate to the closest species or population. Programs for multiple sequence alignment and phylogenetic treebuilding using the aligned sequences and for generating virtual gel for restriction fragment length polymorphism (RFLP) analysis are also available. To support the deposition of new and updated data, a data submission and editing function was created. Users are required to register and $\log$ in to fully access the PD. User authentication is needed to provide a customized means of storing and sharing data via the PD (see below). The cart function allows retrieval of data in the sequence database and also supports data analysis using available tools. Step-by-step guides of how to use available functions were generated using screen shots and can be accessed from multiple locations.

Generation of marker sequences to support sequence-based isolate identification. A key function is supporting the identification of new Phytophthora isolates via comparison of their sequences at one or more loci with the corresponding sequences derived from the reference isolates archived in the PD. To support this func- tion, we have generated and deposited sequence data from more than 1,500 isolates from 83 Phytophthora spp. to the sequence database. Current statistics on the number of species, isolates, and sequences are updated automatically at the PD home page as new data are deposited.

For each isolate, up to nine loci were sequenced, including the following: (i) two loci in the nuclear ribosomal RNA (rRNA) encoding genes: the internal transcribed spacer (ITS) regions and the $5^{\prime}$ portion of the large subunit rRNA gene; (ii) nuclear genes encoding $60 \mathrm{~S}$ ribosomal protein L10, $\beta$-tubulin, enolase, heat shock protein 90, TigA fusion protein, and translation elongation factor 1- $\alpha$ (3); and (iii) a mitochondrially encoded coxII gene and spacer region between coxI and coxII. PCR reaction conditions for amplifying these marker loci, including the sequences and positions of primers used, can be found in the Genetic Markers section and are hyperlinked from marker names throughout the PD user interface. Because ITS sequences commonly have been used for identifying oomycete species and isolates, the ITS region was sequenced from all isolates archived in the PD. The ITS region of an additional 2,000 isolates currently is being sequenced or resulting sequences are being edited. Most sequence data archived in the PD were submitted to GenBank, and a GenBank accession number is shown for each sequence. New sequence data will be submitted periodically to GenBank.

A comprehensive phylogenetic analysis was performed (using Pythium vexans as an outgroup) to establish evolutionary relationships among the known species (3). For this analysis, sequences of seven loci (all the markers described above except the ITS and cox regions) derived from 228 isolates from 83 species were utilized. The result is shown in the form of a genus-wide

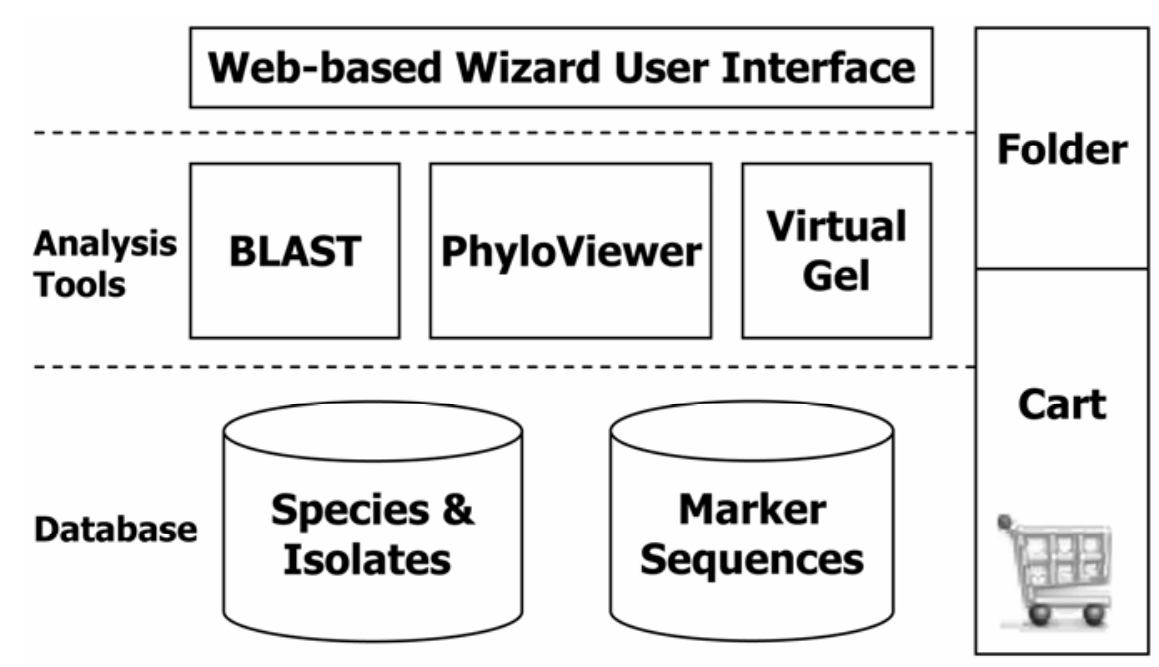

Fig. 1. Organization of the Phytophthora Database (PD). It consists of three main layers, including the user interface (UI), data search and analysis tools, and databases. The UI supports database search, data submission, and navigation through the PD. The folder function allows for the storage and sharing of data, and the cart function supports the retrieval of data from the sequence database and subsequent data analysis. 
phylogenetic tree via individual species pages (see below) and will be updated once new species have been added to the PD. Sequences employed in this analysis are available for downloading.

Database for species and isolate information. This database houses an array of information that will help PD users quickly access characteristics of the archived species and isolates (Fig. 2). When a user selects a species name, the corresponding species information page appears. To provide an evolutionary context, its position in a genus-wide phylogenetic tree is shown at the top. A brief description of species, including nomenclature, morphological and growth characteristics, hosts and disease symptoms, life cycle, known control or management strategies, diagnostic methods, and selected references also is provided. Information for the species page was derived mainly from the following three sources: (i) nomenclature information was created by the United States Department of Agriculture-
Agricultural Research Service Systematic Botany and Mycology Laboratory (additional information, including plant host and geographic distribution, can be found by following the link to the laboratory); (ii) morphological and growth characteristics and a list of known hosts were derived from Phytophthora Diseases Worldwide (10); and (iii) additional information, including diseases pictures, was assembled by individual authors and their collaborators (see Phytophthora ramorum, $P$. infestans, $P$. cinnamomi, $P$. capsici, and $P$. cactorum as examples).

For many species, information from the first two sources is almost complete. However, descriptions of diseases, particularly pictures showing disease symptoms on known hosts, and control or management strategies still remain to be completed for many species. This task requires contributions from members of the global Phytophthora research community; additionally, in order to update the information periodically, their involvement is critical. It is our objective to make the PD a global resource similar to GenBank, through which members of the global Phytophthora research community share and utilize knowledge and data.

At the isolate level, we archived the following information where known: species identity, collector, date and location of isolation, mating type, fungicide resistance, and references. The database automatically generates a unique identification (ID) once a new isolate is entered. This is followed by alternative IDs which designate the sources and accession numbers of the isolate, because it is not uncommon that multiple culture collection facilities archive the same isolate. This is crucial to avoid analysis of the same isolates multiple times and also will guide users to a source for obtaining desired isolates. Available marker sequences for each isolate are shown at the bottom of the isolate page.

User verification. In order to fully utilize the database search and data retrieval

A

B

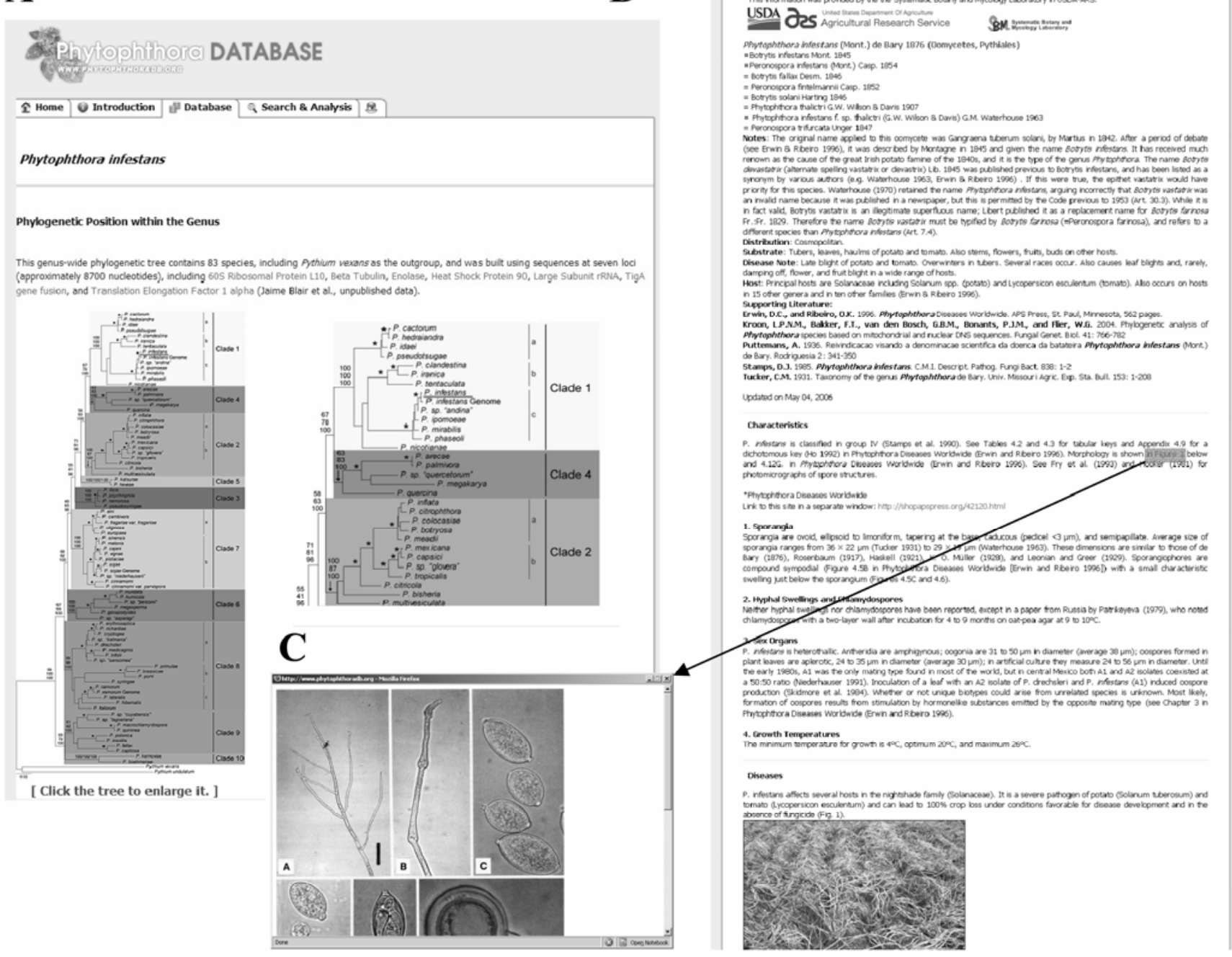

Fig. 2. Species information page for Phytophthora infestans. Parts of the page are shown via screen shots to illustrate the type and organization of available information. A, Position of $P$. infestans in a genus-wide phylogenetic tree (left) with a magnified view (right) showing $P$. infestans and related species. B, Nomenclature information and disease description. C, Not all figures and tables cited in the species page are shown, but can be accessed by clicking their hyperlinked name. Separate window showing morphological features of $P$. infestans is shown. 
functions, users are required to register and $\log$ in. However, some information, such as the user manuals, species information, genetic markers, and references, can be accessed without logging in. A main reason for implementing the user-verification system is to enable customized PD applications. Each user can create private folders for storing results from previous analyses and can share results and sequence data via shared folders. The user can designate users who can access each shared folder by designating user IDs permitted to access each shared folder. This feature enables communication and collaboration among multiple users via the PD. Another reason for user verification is the need for controlled access to sensitive information. It is also undesirable to allow all users the ability to modify stored information. Specific users can be designated curators with the authority to modify stored data. The registration system assigns a level of authority for data access and modification for each user at the time of registration, which can be changed later if necessary.

Data submission. Although we have assembled comprehensive datasets for certain species, covering the entire genus comprehensively is an ongoing task and heavily depends on contribution from members of the global Phytophthora research community. Such contribution also is need to update the content of the PD periodically. To facilitate their contribution, the PD provides multiple mechanisms for new and updated data submission. Species information can be created via a web-based form. In this form, images depicting different stages of the life cycle (e.g., spore morphology and disease symptoms) can be uploaded and linked to the life cycle. Sequence data for individual isolates also can be submitted using the web-based form. To submit data for one or a few isolates, this web-based form should be employed. Researchers who want to submit data for a large number of isolates can use a spreadsheet format to organize the data. The curator of the PD then will upload data in the completed form to the appropriate databases. The curator also can work with those researchers possessing databases in order to develop a customized means for data transfer. Those who submit unpublished sequence data will have an option of making the data unavailable to the general public until publication, a practice adopted from GenBank. However, prompt release of data is encouraged.

Sequence-based database search. Stored sequence data can be searched using a BLAST tool. Data flow through BLAST and two other analysis tools is illustrated in Figure 3, and detailed descriptions of how to use these functions and of how data can be moved from one analysis tool to another can be found in the user manual. The BLAST tool allows for the identification of an unknown isolate by querying the sequence database using one or more of the marker sequences described above. Given the comprehensive set of ITS sequences available for the archived isolates, we suggest that users begin the identification process using this locus, which should establish its identity at or near the species level. If the closest match exhibits substantial sequence differences from the query, it is possible that the unknown isolate may belong to a new species. In the latter case, one may sequence all or some of the seven loci used for the genus-wide phylogenetic analysis (3) to investigate this possibility further. Correct identification of species lineages relies on the analysis of multiple gene phylogenies (32). Sequence alignments and individual sequences can be downloaded to address phylogenetic questions in depth.

In species where many isolates share identical sequences at the ITS region, a BLAST search will retrieve a large number of identical ITS sequences. This makes it difficult for users to know how many unique genotypes exist within a species. To allow users to view all unique genotypes within a species or group of related species without drastically increasing the number of isolates shown in the BLAST output (the default number is 20 , with options ranging from 10 to 50 ), we implemented an automatic process that groups isolates sharing identical sequences. This grouping also helps users analyze unique genotypes in other data analysis environments (Fig. 3 and see below). The grouping process is executed when new data enter the sequence database, which results in either an addition to an existing group or the formation of a new group. Only one isolate representing each group is presented in the BLAST output. However, users can view all isolates in a chosen group using the Group-off option. BLAST results can be saved in a user's folder or moved to a different data analysis tool via the cart function.

Phyloviewer, a new tool for building phylogenetic trees. A suite of tools for building phylogenetic trees, termed Phyloviewer (http://phyloviewer.riceblast.snu. ac.kr/; J. Park, unpublished), was developed to help users visualize evolutionary relationships between a new isolate and related isolates. This tool consists of the ClustalW program (33), a tool for multiple sequence alignment, and a newly developed program that builds a phylogenetic tree using the resulting alignment (Fig. 4)

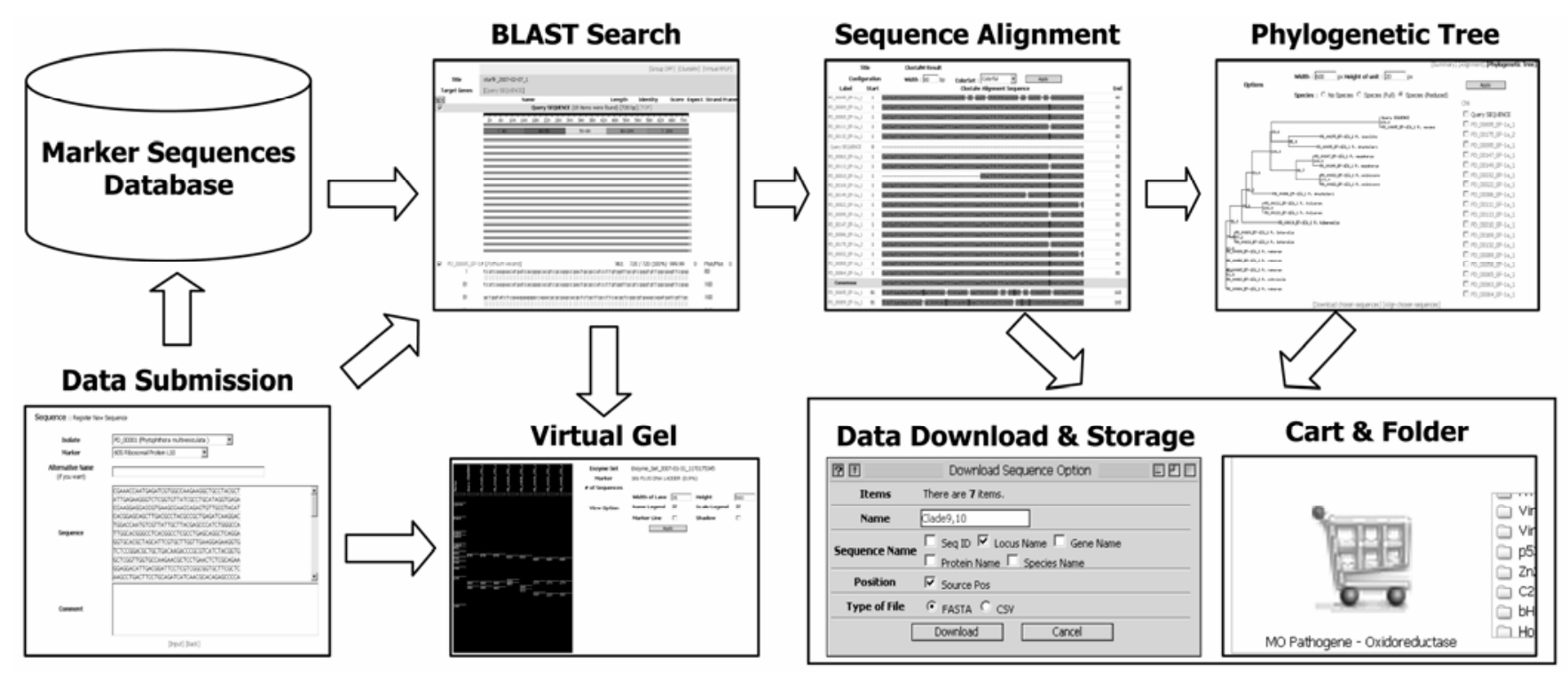

Fig. 3. Data flow via the Phytophthora Database (PD). A marker sequence from a new isolate can be deposited to the PD, used to identify the closest species or isolates via BLAST, or analyzed by the virtual gel tool to view restriction fragment length polymorphism patterns. BLAST results, including the query sequence, can be sent to Phyloviewer or Virtual Gel. Outputs from all analysis tools and individual sequences can be saved in the user's folder or cart in the $\mathrm{PD}$ or downloaded to the user's own computer. 
Users can perform this analysis using sequence data in a BLAST search result and have the option of including all isolates or selected isolates from the BLAST result. Users also can retrieve selected sequences from the sequence database for this analysis. Chosen sequences will be processed in the following order: (i) aligned by ClustalW to create a distance matrix, (ii) converted to a neighbor-joining tree with 1,000 bootstrap replicates, and (iii) transferred to a treedrawing program. Phyloviewer has a number of features that help users view and analyze data in the resulting tree and include the following: (i) the query isolate is labeled with a color different from other isolates, (ii) individual branches are hyperlinked to corresponding groups of isolates, and (iii) isolates from any branch can be moved to different data analysis or visualization environments. Because only one representative isolate for each genotype will be shown, resulting trees will be simple to view. However, if selected, all isolates in each group can be shown.

Although Phyloviewer is useful for quickly visualizing evolutionary relationships among selected isolates, it provides only a crude estimate of phylogenetic relatedness that is unsuitable for publication. Because sequence alignments produced by ClustalW often require manual adjustments prior to building phylogenetic trees, especially when a set of highly divergent sequences are aligned, evolutionary relationships derived from Phyloviewer should be considered tentative. Nondistance-based methods of phylogenetic inference (maximum parsimony, maximum likelihood, and Bayesian) are not currently supported. Users can download a sequence alignment file (e.g., NEXUS format) to adjust the alignment manually and conduct their own phylogenetic analysis using appropriate software packages such as Phylogenetic Analysis Using Parsimony (PAUP; 31) or MEGA (22).

Virtual Gel for RFLP analysis. RFLP analysis of PCR products from certain marker loci, such as the ITS and cox regions, have been utilized as a means for rapid identification of new Phytophthora isolates $(25,26,34)$. To support this diagnostic method, we developed a tool for generating predicted RFLP patterns from user-chosen sequences via a virtual gel. Users can use a BLAST result or sequences retrieved from the sequence database to perform this analysis. Predicted RFLPs with the user-selected restriction enzymes will be shown on a virtual gel along with a size standard (Fig. 5).

\section{DISCUSSION}

The PD cyberinfrastructure was developed to support systematic cataloging and utilization of a diverse array of data associated with Phytophthora spp. and isolates so that members of the global Phytophthora research community can easily access, update, analyze, and share data via the web. Because the PD provides applied and molecular information on Phytophthora spp. with an integrated suite of data analysis tools, we believe the PD has more utility than GenBank. In addition, the data stored in the PD are provided by specialists actively working with Phytophthora spp.; hence, proper identification of reference cultures should be less prone to error. Many data are connected to vouchered cultures available to appropriately permitted researchers.

We had three objectives in building the PD. Our first and foremost aim was to collect and catalog Phytophthora-related data and materials in a format that maximally supports their present and future uses for understanding the taxonomy and ecology of Phytophthora spp. Our second aim was to promote and facilitate data integration and sharing. Because science builds on existing knowledge, we cannot overemphasize the importance of properly archiving accumulated data and materials in such a format (21). Collecting data without the consideration of subsequent integration with other data sets from the scientific community impedes the utiliza-

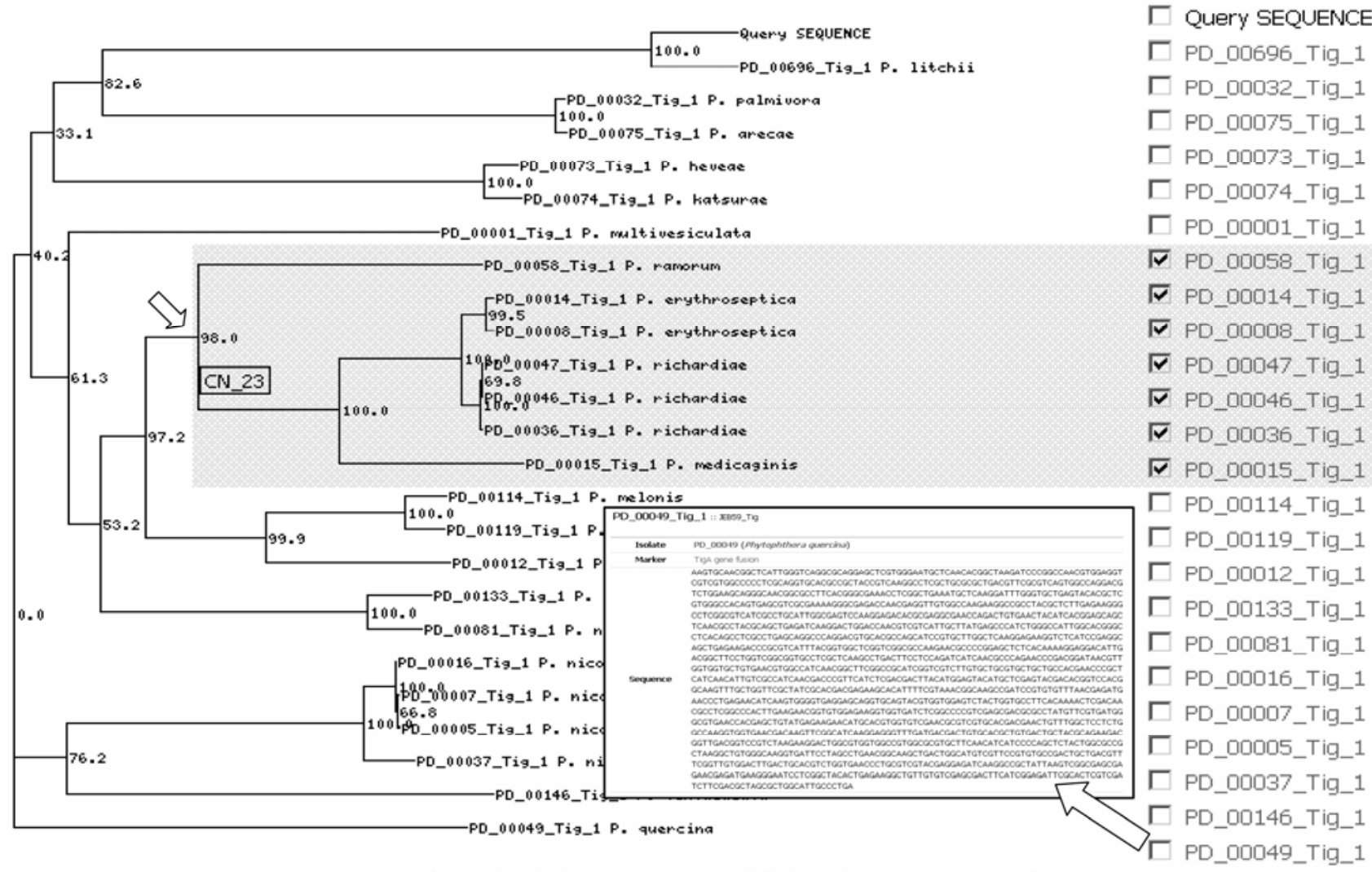

[Download chosen sequences] [Align chosen sequences]

Fig. 4. Phylogenetic tree built from Phyloviewer. Sequences aligned by ClustalW are converted to a neighbor-joining tree with bootstrap replicates. The chosen marker sequence for each of the isolates in this tree can be viewed in a separate window by clicking their identifications (IDs). Selected sequences (by checking the box next to ID or clicking a node of the tree) can be downloaded, realigned for building a new tree, or moved to different data analysis environments via the cart function. 
tion of that data to create a global picture. The amassing of data without the establishment of proper integration, interpretation, and analysis tools will result in the state of being data rich but knowledge poor. In particular, the sharing of knowledge with the international plant pathology community is critical for protection against invasive pathogens. Because many highrisk pathogens $(17,24)$ currently are not present in the United States, we do not have adequate knowledge of their biology, ecology, and epidemiology. Addressing this deficiency is a difficult task, because research on such topics is severely restricted in the United States due to the requirement of high-level containment facilities and strict regulatory compliance. This quandary highlights the importance of cooperation with scientists in countries where target pathogens may be endemic and detailed information on the pathogens has been accumulated.

Our third aim was to maximize the benefits from Phytophthora genomics. Three Phytophthora genomes have been sequenced $(P$. sojae, $P$. infestans, and $P$. ramorum), and the genome sequence of $P$. capsici is nearly completed $(15,35)$. Realizing the full potential of Phytophthora genomics as a foundation for understanding and managing Phytophthora diseases

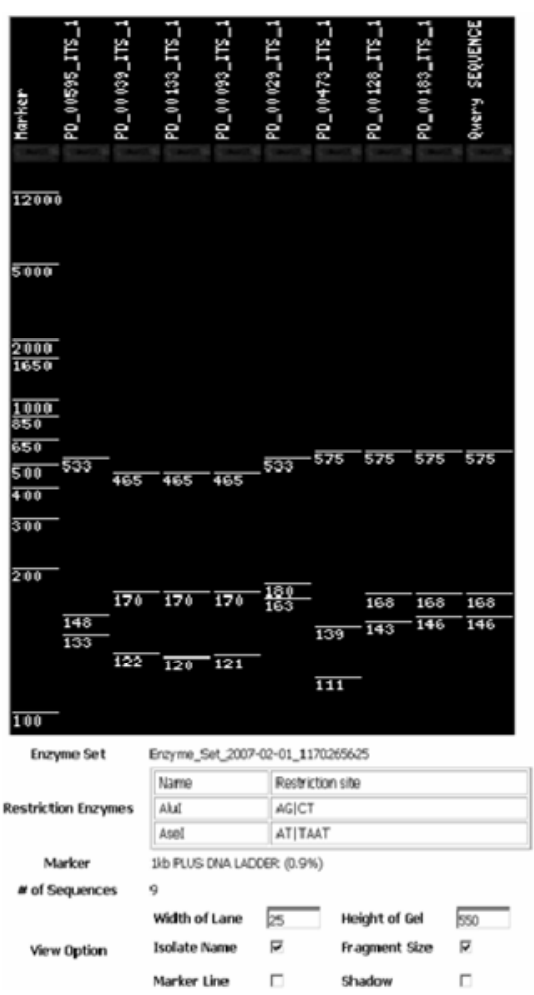

Fig. 5. Virtual Gel. To generate restriction fragment length polymorphisms for chosen sequences, users need to first select a restriction enzyme or a combination of restriction enzymes from the menu. Users can specify the format and information content of a gel by choosing a number of options, including the width of individual lanes, the height of the gel, and the sizes of the restriction fragments. relies on how effectively we use genome sequences and data from gene functional studies to gain a better understanding of genotypic and phenotypic diversity and the evolutionary potential within a species; this requires a collection of diverse isolates with adequate biological information.

We hope to establish the PD as a global "e-research and monitoring forum," through which scientists worldwide will be able to access, contribute, and analyze data on Phytophthora diseases. This forum would provide the best available information to quickly assess the risk of a new or emerging Phytophthora sp. and make decisions regarding how the situation should be addressed. The genus-wide phylogeny (3), based on reference cultures and multiple marker genes, should facilitate the identification and classification of unknown isolates and provide a framework that illustrates the genus-wide diversity and evolutionary relationships. These data also will aid the development and validation of molecular diagnostic protocols. Diagnosis-based on genetic markers unique to a target pathogen is a highly effective tool for detection because of increased sensitivity and selectivity. However, without a comprehensive understanding of the genetic diversity in the genus Phytophthora, molecular diagnostic approaches designed for species-specific identification cannot be fully validated. Although a significant amount of data has been deposited and a number of data analysis and visualization tools are in place, the current form is not the final product. Sequence and morphological data from new species and sequence data from isolates from contemporary field surveys will be continually generated. Because many of the genetic markers used in our study correspond to relatively wellconserved genes, they may not be suitable for characterizing species complexes whose members likely exhibit similar morphology but consist of distinct phylogenetic species $(13,32)$. As such, we have begun to develop new markers suitable for such analyses. Thanks to increasing genome sequence and expressed sequence tag data, this task is becoming easier. Validated makers will be posted on the genetic markers database.

Because one of our future goals is to incorporate Geographic Information System tools into the PD, so that the origins of chosen isolates can be viewed across geospatial and temporal scales in the context of environmental data, we need data derived from isolates with accurate geospatial and temporal contexts. Unfortunately, many historical isolates archived in the PD lack detailed information on their geographic origin; typically, information ends at the country or state or province level. Visualization of the geographic origins of strains genetically related to a new isolate, in the form of a map with zoom function, will help identify the likely origin of the new isolate. Although it has been several years since $P$. ramorum was first identified in both the United States and Europe $(29,36)$, its origin remains unknown. If comprehensive data on the genetic diversity of Phytophthora spp. existed on a global scale prior to the outbreak of this disease, subsequent detective work might have been much easier. A better understanding of pathogen dynamics in geospatial and temporal contexts also may provide insights into the mechanisms underpinning changes in pathogen communities.

Published markers for detection of specific species will be presented to support molecular diagnosis,. To facilitate the development of markers for additional species, the sequence alignments of regions used for marker development also will be presented. In addition, sequencing of mitochondria genomes for a number of species is in progress and will provide sequence data that will be useful for species identification as well as individual mitochondrial haplotypes within individual species.

To facilitate future expansions of the $\mathrm{PD}$, establishing a cohesive computational environment that will seamlessly support the movement of data from one analysis environment to another has been the guiding principle in developing the PD. The current and future tools in the PD provide a powerful means to analyze and visualize the structure and dynamics of Phytophthora spp. However, their utility will be diminished significantly without continuous data deposition and regular updates. As such, we need to strengthen ties with international plant pathologists and urge members of the global Phytophthora community to share their knowledge and data through the PD.

In the long run, we hope that the impact of the PD reaches far beyond a better understanding of Phytophthora spp. and their management. The PD platform easily can be adopted, with minimal modification, to support cataloging and sharing different important pathogen groups, so that researchers and regulators worldwide could work more closely and make better use of data available. All tools and database designs described in this paper are free to others interested in projects requiring similar informatics tools.

\section{ACKNOWLEDGMENTS}

This project has been supported by grants from the USDA-NRI Plant Biosecurity program (200535605-15393) and the Pennsylvania Department of Agriculture (ME442316 and ME 445580), and a cooperative agreement with USDA-ARS (59-19203-304). Y.-H. Lee acknowledges a grant from the Korean Research Foundation (KRF-2006-005J04701). Assistantships for J. Park and K. Jung were provided by the Brain Korea 21 Project. We are deeply grateful to the American Phytopathological Society Press for permitting us to use parts of the book Phytophthora Diseases Worldwide. We thank G. Abad (USDA-APHIS-PPQ- 
PHP-PSPI-MDL), D. E. L. Cooke (Scottish Crop Research Institute), and T. Oszako (Department of Forest Phytopathology, Raszyn, Poland) for providing cultures representing a few species.

\section{LITERATURE CITED}

1. Agrios, G. N. 2005. Plant Pathology. Academic Press, San Diego, CA.

2. Altschul, S. F., Gish, W., Miller, W., Myers, E. W., and Lipman, D. J. 1990. Basic local alignment search tool. J. Mol. Biol. 215:403410.

3. Blair, J. E., Coffey, M. D., Park, S., Geiser, D. M., and Kang, S. 2008. A multi-locus phylogeny for Phytophthora utilizing markers derived from complete pathogen genomes. Fungal Genet. Biol. 45:266-277.

4. Bonants, P. J. M., Hagenaar-de Weerdt, M., Man in 't Veld, W. A., and Baayen, R. P. 2000. Molecular characterization of natural hybrids of Phytophthora nicotianae and P. cactorum. Phytopathology 90:867-874.

5. Brasier, C. M., Beales, P. A., Kirk, S. A., Denman, S., and Rose, J. 2005. Phytophthora kernoviae sp. nov., an invasive pathogen causing bleeding stem lesions on forest trees and foliar necrosis of ornamentals in the UK. Mycol. Res. 109:853-859.

6. Brasier, C. M., Cooke, D. E. L., and Duncan, J. M. 1999. Origin of a new Phytophthora pathogen through interspecific hybridization. Proc. Natl. Acad. Sci. USA 96 (10):5878-5883.

7. Brasier, C. M., Cooke, D. E. L., Duncan, J. M., and Hansen, M. E. 2003. Multiple new phenotypic taxa from trees and riparian ecosystems in Phytophthora gonapodyides-P. megasperma ITS clade 6 , which tend to be high-temperature tolerant and either inbreeding or sterile. Mycol. Res. 107:277-290.

8. Brasier, C. M., Kirk, S. A., Delcan, J., Cooke, D. E. L., Jung, T., and Man in 't Veld, W. A. 2004. Phytophthora alni sp. nov. and its variants: designation of emerging heteroploid hybrid pathogens spreading on Alnus trees. Mycol. Res. 108:1172-1184

9. Cooke, D. E. L., Jung, T., Williams, N. A., Drenth, A., Schubert, R., Oßward, W., and Duncan, J. M. 2005. Genetic diversity of European populations of the oak fine-root pathogen Phytophthora quercina. For. Pathol. 35:57-70.

10. Erwin, D. C., and Ribeiro, O. K. 1996. Phytophthora Diseases Worldwide. American Phytopathological Society Press, St. Paul, MN.

11. Flier, W. G., Grünwald, N. J., Kroon, L. P. N. M., van den Bosch, T. B. M., Garay-Serrano, E., Lozoya-Saldaña, H., Bonants, P. J. M., and Turkensteen, L. J. 2002. Phytophthora ipomoeae sp. nov., a new species causing leaf blight on Ipomoea longipedunculata in the Toluca Valley of central Mexico. Mycol. Res. 106:848-856.

12. Fry, W. E., and Goodwin, S. B. 1997. Reemergence of potato and tomato late blight in the United States. Plant Dis. 81:1349-1357.

13. Geiser, D. M. 2004. Practical fungal molecular taxonomy. Pages 1-12 in: Advances in Fungal Biotechnology for Industry. J. Tkacz and L.
Lange, eds. Kluwer Academic Publishers, Dordrecht, The Netherlands.

14. Gibbs, J. N. 1978. Intercontinental epidemiology of Dutch elm disease. Annu. Rev. Phytopathol. 16:287-307.

15. Govers, F., and Gijzen, M. 2006. Phytophthora genomics: The plant destroyers' genome decoded. Mol. Plant-Microbe Interact. 19:12951301.

16. Hansen, E. M., Reeser, P., Davidson, J. M., Garbelotto, M., Ivors, K., Douhan, L., and Rizzo, D. M. 2003. Phytophthora nemorosa, a new species causing cankers and leaf blight of forest trees in California and Oregon. Mycotaxon 88:129-138.

17. Hawks, B. 2002. Agricultural Bioterrorism Protection Act of 2002; listing of biological agents and toxins and requirements and procedures for notification of possession. Fed. Regist. 67:52383-52389.

18. Jung, T., Cooke, D. E. L., Blaschke, H., Duncan, J. M., and Oßwald, W. F. 1999. Phytophthora quercina sp. nov., causing root rot of European oaks. Mycol. Res. 103:785-798.

19. Jung, T., Hansen, E. M., Winton, L., Oßwald, W. F., and Delatour, C. 2002. Three new species of Phytophthora from European oak forests. Mycol. Res. 106:397-411.

20. Jung, T., Nechwatal, J., Cooke, D. E., Hartman, G., Blaschke, M., Oswald, W. F., Duncan, J. M., and Delatour, C. 2003. Phytophthora pseudosyringae sp. nov., a new species causing root and collar rot of deciduous tree species in Europe. Mycol. Res. 107:772-789

21. Kang, S., Blair, J. E., Geiser, D. M., Khang, C., Park, S., Gahegan, M., O'Donnell, K., Luster, D. G., Ivors, K. I., Kim, S. H., Lee, Y., Lee, Y., Grünwald, N. J., Martin, F. M., Coffey, M. D., Veeraraghavan, N., and Makalowska, I. 2006. Plant pathogen culture collections: it takes a village to preserve these resources vital to the advancement of agricultural security and plant pathology. Phytopathology 96:920-925.

22. Kumar, S., Tamura, K., and Nei, M. 2004. MEGA3: Integrated software for molecular evolutionary genetics analysis and sequence alignment. Brief. Bioinf. 5:150-163.

23. Liebhold, A. M., MacDonald, W. L., Bergdahl, D., and Mastro, V. C. 1995. Invasion by exotic forest pests: A threat to forest ecosystems. For. Sci. Monogr. 30:1-49.

24. Madden, L. V., and Wheelis, M. 2003. The threat of plant pathogens as weapons against U.S. crops. Annu. Rev. Phytopathol. 41:155176.

25. Martin, F. N., and Tooley, P. W. 2004. Identification of Phytophthora isolates to species level using RFLP analysis of a PCR amplified region of mitochondrial DNA. Phytopathology 94:983-991.

26. Martin, F. N., Tooley, P. W., and Blomquist, C. 2004. Molecular detection of Phytophthora ramorum, the causal agent of sudden oak death in California, and two additional species commonly recovered from diseased plant material. Phytopathology 94:621-631.
27. Park, J., Park, B., Jung, K., Jang, S., Yu, K., Choi, J., Kong, S., Park, J., Kim, S., Kim, H., Kim, S., Kim, J., Blair, J., Lee, K., Kang, S. and Lee, Y.-H. 2008. CFGP: A web-based, comparative fungal genomics platform. $\mathrm{Nu}$ cleic Acids Res. 36:D562-D571.

28. Polashock, J. J., Vaiciunas, J., and Oudemans, P. V. 2005. Identification of a new Phy tophthora species causing root and runner rot of cranberry in New Jersey. Phytopathology 95:1237-1243.

29. Rizzo, D. M., Garbelotto, M., Davidson, J. M., Slaughter, G. W., and Koike, S. T. 2002. Phytophthora ramorum as the cause of extensive mortality of Quercus spp. and Lithocarpus densiflorus in California. Plant Dis. 86:205214.

30. Rizzo, D. M., Garbelotto, M., and Hansen, E. M. 2005. Phytophthora ramorum: Integrative research and management of an emerging pathogen in California and Oregon forests. Annu. Rev. Phytopathol. 43:309-335.

31. Swofford, D. L. 2002. PAUP*: Phylogenetic Analysis Using Parsimony (and Other Methods), 4.0 beta. Sinauer and Associates, Sunderland, MA.

32. Taylor, J. W., Jacobson, D. J., Kroken, S., Kasuga, T., Geiser, D. M., Hibbett, D. S., and Fisher, M. C. 2000. Phylogenetic species recognition and species concepts in fungi. Fungal Genet. Biol. 31:21-32.

33. Thompson, J. D., Higgins, D. G., and Gibson, T. J. 1994. The ClustalW: Improving the sensitivity of progressive multiple sequence alignment through sequence weighting, position, specific gap penalties, and weight matrix choice. Nucleic Acids Res. 22:4673-4680.

34. Tripathi, A., Singh, R., Raj, S. K., Singh, A. P., and Johri, J. K. 2003. Molecular identification of Phytophthora nicotianae isolates causing leaf rot of betelvine (Piper betle L.). Curr. Sci. 84:22-24.

35. Tyler, B. M., Tripathy, S., Zhang, X., Dehal, P., Jiang, R. H. Y., Aerts, A., Arredondo, F. D., Baxter, L., Bensasson, D., Beynon, J. L., Chapman, J., Damasceno, C. M. B., Dorrance, A. E., Dou, D., Dickerman, A. W., Dubchak, I L., Garbelotto, M., Gijzen, M., Gordon, S. G. Govers, F., Grunwald, N. J., Huang, W., Ivors, K. L., Jones, R. W., Kamoun, S., Krampis, K., Lamour, K. H., Lee, M.-K., McDonald, W. H. Medina, M., Meijer, H. J. G., Nordberg, E. K. Maclean, D. J., Ospina-Giraldo, M. D., Morris, P. F., Phuntumart, V., Putnam, N. H., Rash, S., Rose, J. K. C., Sakihama, Y., Salamov, A. A., Savidor, A., Scheuring, C. F., Smith, B. M., Sobral, B. W. S., Terry, A., Torto-Alalibo, T. A., Win, J., Xu, Z., Zhang, H., Grigoriev, I. V., Rokhsar, D. S., and Boore, J. L. 2006. Phytophthora genome sequences uncover evolutionary origins and mechanisms of pathogenesis. Science 313:1261-1266.

36. Werres, S., Marwitz, R., Man in 't Veld, W. A., De Cook, A. W. A. M., Bonants, P. J. M., De Weerdt, M., Themann, K., Ilieva, E., and Baayen, R. P. 2001. Phytophthora ramorum sp. nov., a new pathogen on Rhododendron and Viburnum. Mycol. Res. 105:1155-1165. 